\title{
SPEECH PATHOLOGY FINDINGS IN PATIENTS WITH ORAL BREATHING ACCORDING TO THE ETIOLOGY
}

\author{
P. Junqueira ${ }^{1}$ I.Q. Marchesan $^{2}$ \\ ${ }^{1}$ Care Center for Oral Breather, ${ }^{2}$ Speech Disorders, CEFAC, Sao Paulo, Brazil
}

Some authors claim that alterations in speech, language, voice, chewing and deglutition are characteristics of oral breathing children. Because it is a symptom and it has causes, that many times are multifactorial, it is necessary to clarify more details about the clinical aspects of this entity. This study aimed to identify and compare the findings of alterations in: speech; orofacial functions; positions of lips and tongue; voice and language in children, 2 to 16 year old, with a history of oral breathing, according to the following diagnoses: allergic rhinitis; adenoidal hypertrophy; allergic rhinitis and adenoidal and functional hypertrophy. 414 patients of both genders, from 2 to 16 year old, of the Care Center for Oral Breather were evaluated by a team made up by speech pathologists, allergists, and otolaryngologists who carried out clinical and integrated examinations (CBC, X-ray, nasal endoscopy, audiometry) of all patients. The diagnosis of oral breathing etiology was based on specific serum measurements of IgE (imunoglobolineE), X-ray of paranasal sinuses and/or nasal endoscopy. Data were statistically analyzed. The patients were divided according to otologic diagnosis for oral breathing. The etiology, most present in the sample, was allergic rhinitis. The most observed speech-language diagnosis was the presence of alterations in orofacial functions; position of lips and tongue and tone, separately or accompanied by other alterations. The studied sample showed presence of $82.2 \%$ relative to speech-language alterations. There were no statistically significant associations between the etiology of the patients and presence and/or number of alterations in speech-language diagnosis. 